\title{
CLASSIFICATION OF NORMAL SUBGROUPS OF THE MODULAR GROUP
}

\author{
BY \\ MORRIS NEWMAN
}

1. Introduction. Let $\Gamma$ be the modular group, consisting of all linear fractional transformations

$$
\tau^{\prime}=(a \tau+b) /(c \tau+d)
$$

where $a, b, c, d$ are rational integers and $a d-b c=1$. Then $\Gamma$ is generated by the linear fractional transformations $S, T$ where $S \tau=\tau+1, T \tau=-1 / \tau$ and is the free product of the cyclic group $\{T\}$ of order 2 and the cyclic group $\{S T\}$ of order 3 . Let $G$ be a normal subgroup of $\Gamma$ of finite index $\mu$. It is known that there is just one such subgroup for $\mu=1,2,3$ which may be described as $\Gamma^{\mu}$, the subgroup of $\Gamma$ generated by the $\mu$ th powers of the elements of $\Gamma$. In all other cases $\mu$ must be a multiple of 6 (see [3] or [6]) and there are only finitely many normal subgroups of $\Gamma$ of a given finite index $\mu$, since the total number of subgroups of a given finite index in a finitely generated group is finite.

The purpose of this article is to obtain some information about the function $N(\mu)$, the number of normal subgroups of $\Gamma$ of index $\mu$. By the remarks above $N(1)=N(2)=N(3)=1$, and $N(\mu)=0$ if $\mu>3$ and $\mu \neq \equiv(\bmod 6)$. We will determine all normal subgroups of $\Gamma$ for $\mu \leqq 66$, and we will also determine $N(\mu)$ explicitly when $\mu=6 q$ or $12 q$, where $q$ is a prime. For example it will be shown that $N(6 q)$ $=1+(q / 3)$ for all primes $q>3$, and that $N(12 q)=0$ for all primes $q>11$. Here $(q / 3)$ is the Legendre-Jacobi symbol of quadratic reciprocity.

Some recent work of I. M. S. Dey [2] implies that the total number $M(\mu)$ of subgroups of $\Gamma$ of index $\mu$ satisfies the recurrence formula

$$
M(\mu)=\mu \alpha_{\mu}-\sum_{k=1}^{\mu-1} \alpha_{\mu-k} M(k), \quad \mu>1 .
$$

Here $\alpha_{k}=\tau_{2}(k) \tau_{3}(k) / k$ ! and $\tau_{p}(k)$ is the number of homomorphisms of the cyclic group of order $p$ into $S_{k}$, given explicitly for prime $p$ by the formula

$$
\tau_{p}(k)=\sum_{0 \leqq r \leqq k / p} \frac{k !}{r !(k-r p) ! p^{r}} .
$$

The first few values of $M(\mu)$ are given below:

$$
\begin{array}{rrrrrrrrrr}
\mu & 1 & 2 & 3 & 4 & 5 & 6 & 7 & 8 & 9 \\
M(\mu) & 1 & 1 & 4 & 8 & 5 & 22 & 42 & 40 & 120
\end{array}
$$

Received by the editors May 4, 1966. 
No such recurrence formula for $N(\mu)$ is known, however. It would be highly desirable to find such a formula.

We assume from now on that $G$ is a normal subgroup of $\Gamma$ of finite index $\mu>3$, so that $\mu$ is a multiple of 6 . Let $n$ denote the exponent of $S$ modulo $G$ : that is, $n$ is the least positive integer such that $S^{n} \tau=\tau+n$ belongs to $G$. Then $G$ is said to be of level $n$, and $n$ certainly divides $\mu$. We set

$$
t=\mu / n \text {. }
$$

The number $t$ is just the number of parabolic classes of $G$ (see [4]). The genus $g$ of $G$ is then given by

$$
g=1+\frac{\mu}{12}-\frac{t}{2}=1+\mu \frac{n-6}{12 n}
$$

(see [3]).

For each $g \neq 1$ there are only finitely many normal subgroups $G$ of genus $g$. For example the only normal subgroups of genus 0 are $\Gamma, \Gamma^{2}, \Gamma^{3}, \Gamma(2), \Gamma(3), \Gamma(4), \Gamma(5)$. Here for each positive integer $m, \Gamma(m)$ is the principal congruence subgroup of level $m$, consisting of all elements $(1)$ of $\Gamma$ such that $a \equiv d \equiv \pm 1(\bmod m), b \equiv c \equiv 0$ $(\bmod m)$.

The normal subgroups of genus 1 (which are all of level 6) have been completely described by the author in [5]. Let $\Gamma^{\prime}$ denote the commutator subgroup of $\Gamma$. Then $\Gamma^{\prime}$ is of index 6 in $\Gamma$ and is a free group of rank 2, freely generated by

$$
A=S T S^{-1} T, \quad B=T S^{-1} T S .
$$

For every word $W$ of $\Gamma^{\prime}$ let $e_{A}(W), \dot{e_{B}}(W)$ denote the sum of the exponents of $A$ and $B$, respectively. Then $G$ is a normal subgroup of genus 1 if and only if integers $p, m, d$ exist such that

$$
p>0, \quad 0 \leqq m \leqq d-1, \quad m^{2}+m+1 \equiv 0 \quad(\bmod d) ;
$$

and $G$ consists of all words $W$ of $\Gamma^{\prime}$ satisfying

$$
e_{A}(W) \equiv 0 \quad(\bmod p), \quad e_{B}(W) \equiv m e_{A}(W) \quad(\bmod d p) .
$$

The group $G$ is then of index $6 d p^{2}$ in $\Gamma$ and has $d p^{2}$ parabolic classes. We denote this group by $(p, m, d)$.

We put

$$
H=\{S, G\}=G+S G+\cdots+S^{n-1} G .
$$

Thus $(\Gamma: H)=t$, but $H$ is not in general a normal subgroup of $\Gamma$.

If $A_{1}, A_{2}, \ldots$ are elements of $\Gamma$ then $\Delta\left(A_{1}, A_{2}, \ldots\right)$ will stand for the normal closure in $\Gamma$ of $A_{1}, A_{2}, \ldots$ : that is, the intersection of all normal subgroups of $\Gamma$ containing $A_{1}, A_{2}, \ldots$ Since $\Gamma=\{T\} *\{S T\}$, the quotient group $\Gamma / \Delta\left(A_{1}, A_{2}, \ldots\right)$ may be described as the group generated by the symbols $S, T$ with defining relations

$$
T^{2}=(S T)^{3}=A_{1}=A_{2}=\cdots=1
$$


In what follows we make free use of the relations

$$
T^{2}=(S T)^{3}=1,
$$

occasionally in the form

$$
T=T^{-1}, \quad T S T=S^{-1} T S^{-1} .
$$

\section{Preliminary lemmas and theorems.}

LEMMA 1. Suppose that $1 \leqq k \leqq 5$. Let $F_{k}$ be the group generated by $x, y$ with defining relations

$$
x^{2}=(x y)^{3}=1, \quad x y^{k}=y^{k} x .
$$

Then $F_{k}$ is a finite group of order $O_{k}$ and the central element $y^{k}$ has period $e_{k}$, where $e_{k}, O_{k}$ are given in the following table:

$\begin{array}{rrrrrr}k & 1 & 2 & 3 & 4 & 5 \\ e_{k} & 6 & 3 & 4 & 6 & 12 \\ O_{k} & 6 & 18 & 48 & 144 & 720\end{array}$

This result is well known in the theory of the polyhedral groups. See the book by Coxeter and Moser [1, pp. 76-79].

An easy generalization follows:

LEMMA 2. Suppose that $1 \leqq k \leqq 5$. Let $F_{k, m}$ be the group generated by $x, y$ with defining relations

$$
x^{2}=(x y)^{3}=y^{m k}=1, \quad x y^{k}=y^{k} x
$$

where $m \mid e_{k}$. Then $F_{k, m}$ is of order $m O_{k} / e_{k}$.

Proof. Let $\Delta_{m}$ be the normal closure in $F_{k}$ of $y^{m k}$. Then

$$
F_{k, m} \cong F_{k} / \Delta_{m}
$$

Since $y^{k}$ is in the center of $F_{k}, \Delta_{m}$ is just the cyclic group of order $e_{k} / m$ generated by $y^{m k}$. Also $F_{k}$ is of order $O_{k}$. The order of $F_{k, m}$ is thus

$$
\frac{O_{k}}{e_{k} / m}=m O_{k} / e_{k}
$$

and the lemma is proved.

From this lemma we obtain the following theorem concerning normal subgroups of $\Gamma$ :

THEOREM 1. Let $k, m$ be as defined in Lemma 2 above, and put

$$
G_{k, m}=\Delta\left(S^{m k}, T S^{k} T S^{-k}\right) .
$$


Then $G_{k, m}$ is a normal subgroup of $\Gamma$ of finite index $\mu=m O_{k} / e_{k}$ and level $n=m k$. The values of $\mu, n, t, g$ for these groups are given by the table below:

$$
\begin{array}{rrrrrrrrrrrrrrrrrrrr}
k & 1 & 1 & 1 & 1 & 2 & 2 & 3 & 3 & 3 & 4 & 4 & 4 & 4 & 5 & 5 & 5 & 5 & 5 & 5 \\
m & 1 & 2 & 3 & 6 & 1 & 3 & 1 & 2 & 4 & 1 & 2 & 3 & 6 & 1 & 2 & 3 & 4 & 6 & 12 \\
\mu & 1 & 2 & 3 & 6 & 6 & 18 & 12 & 24 & 48 & 24 & 48 & 72 & 144 & 60 & 120 & 180 & 240 & 360 & 720 \\
n & 1 & 2 & 3 & 6 & 2 & 6 & 3 & 6 & 12 & 4 & 8 & 12 & 24 & 5 & 10 & 15 & 20 & 30 & 60 \\
t & 1 & 1 & 1 & 1 & 3 & 3 & 4 & 4 & 4 & 6 & 6 & 6 & 6 & 12 & 12 & 12 & 12 & 12 & 12 \\
g & 0 & 0 & 0 & 1 & 0 & 1 & 0 & 1 & 3 & 0 & 2 & 4 & 10 & 0 & 5 & 10 & 15 & 25 & 55
\end{array}
$$

Proof. The proof is immediate from the remark that

$$
\Gamma / G_{k, m} \cong F_{k, m} .
$$

We return now to the normal subgroup $G$.

LEMMA 3. There is a $k$ such that $1 \leqq k \leqq t, k \mid n$ and

$$
T S^{k} \equiv S^{k} T(\bmod G) .
$$

Furthermore the group $\left\{S^{k}, G\right\}$ is a normal subgroup of $\Gamma$ of index $k t$, level $k$ and having $t$ parabolic classes. In addition if $t>3$ then

$$
k t \equiv 0 \quad(\bmod 6), \quad t \equiv k t / 6 \quad(\bmod 2) .
$$

Proof. The $t+1$ elements $(T S T)^{l}=T S^{l} T, 0 \leqq l \leqq t$ cannot all be distinct modulo $H$ since $(\Gamma: H)=t$. Hence there is a $k$ (which we choose least positive) such that $T S^{k} T \in H$, and $1 \leqq k \leqq t$. It follows that there is an $\alpha$ such that $0 \leqq \alpha \leqq n-1$ and

$$
(T S T)^{k} \equiv S^{\alpha} \quad(\bmod G) .
$$

Hence

$$
\left(S^{-1} T S^{-1}\right)^{k} \equiv S^{\alpha} \quad(\bmod G),
$$

and conjugating by $S$ we find that

$$
\left(T S^{-2}\right)^{k} \equiv S^{\alpha} \quad(\bmod G) .
$$

Now conjugating separately by $T$ and by $S^{-2}$ we find that

$$
\left(S^{-2} T\right)^{k} \equiv T S^{\alpha} T \equiv S^{\alpha} \quad(\bmod G)
$$

which implies that

$$
T S^{k} \equiv S^{k} T(\bmod G) .
$$

The fact that $k \mid n$ follows from the fact that $T S^{n} T$ also belongs to $H$ and that $k$ was least positive. 
Consider now the group

$$
G^{*}=\left\{S^{k}, G\right\}=G+S^{k} G+\cdots+S^{k(n / k-1)} G .
$$

Since $S^{k}$ commutes with $S$ and $T$ modulo $G$ and $S, T$ are generators of $\Gamma, G^{*}$ is also a normal subgroup of $\Gamma$. Let $\mu^{*}, n^{*}, t^{*}, g^{*}$ denote the index, level, parabolic class number and genus respectively of $G^{*}$. Since $\left(G^{*}: G\right)=n / k$, we find that

$$
\mu^{*}=k t, \quad n^{*}=k, \quad t^{*}=t, \quad g^{*}=1+t(k-6) / 12 .
$$

The congruences (6) now follow from the remarks that if $\mu^{*}>3$ then $\mu^{*} \equiv 0$ (mod 6), and that $g^{*}$ is an integer. This concludes the proof of the lemma.

We also require

LeMma 4. Suppose that

$$
T S^{6} \equiv S^{6} T(\bmod G) .
$$

Then there is a $k$ such that $1 \leqq k \leqq t$, and $n \mid 12 k$.

Proof. By the argument used in the proof of Lemma 3, there is a $k$ such that $1 \leqq k \leqq t$ and $\left(T S^{-3}\right)^{k} \in H$. Hence for some $\alpha$ satisfying $0 \leqq \alpha \leqq n-1$,

$$
\left(T S^{-3}\right)^{k} \equiv S^{\alpha} \quad(\bmod G)
$$

Conjugating by $S^{-3}$, we also have

$$
\left(S^{-3} T\right)^{k} \equiv S^{\alpha} \quad(\bmod G)
$$

Now $T S^{-3}$ and $S^{-3} T$ commute modulo $G$, because of (7). It follows that

$$
S^{-6 \kappa} \equiv S^{2 \alpha} \quad(\bmod G)
$$

so that

$$
\left(T S^{-3}\right)^{2 k} \equiv S^{-6 k} \quad(\bmod G)
$$

Conjugating by $S^{-1}$,

$$
\begin{aligned}
\left(S^{-1} T S^{-2}\right)^{2 k} & \equiv S^{-6 k} \quad(\bmod G), \\
\left(T S T S^{-1}\right)^{2 k} & \equiv S^{-6 k} \quad(\bmod G) .
\end{aligned}
$$

Put $C=T S T S^{-1}$. Then $C^{-1}=T C T$. This implies that

$$
\begin{aligned}
S^{6 k} & \equiv T S^{-6 k} T \equiv S^{-6 k} \quad(\bmod G), \\
S^{12 k} & \equiv 1 \quad(\bmod G) .
\end{aligned}
$$

Hence $n \mid 12 k$ and the lemma is proved.

Some corollaries follow.

Corollary 1. Suppose that $(n, t !)=1$. Then $n \mid 6$.

Proof. Lemma 3 implies that $T S \equiv S T(\bmod G)$. Lemma $1($ for $k=1)$ now implies that $n \mid 6$. 
Corollary 2. Suppose that $1 \leqq t \leqq 6$. Then $n \mid n_{t}$, where $n_{t}$ is given in the following table:

$\begin{array}{rrrrrrr}t & 1 & 2 & 3 & 4 & 5 & 6 \\ n_{t} & 6 & 6 & 12 & 24 & 120 & 720\end{array}$

Proof. For $1 \leqq t \leqq 5$, the corollary follows from Lemmas 1 and 3 by choosing $n_{t}$ as the least common multiple of $k e_{k}$ for $1 \leqq k \leqq t$. For $t=6$ the corollary follows similarly from Lemmas 1,3 and 4 .

3. The principal theorems. The preceding lemmas and corollaries imply the following interesting result:

THEOREM 2. There are only finitely many normal subgroups of $\Gamma$ having at most 11 parabolic classes.

Proof. If $t \leqq 6$ then there are only finitely many possible values for $t$ and for $n$, by Corollary 2 ; hence for $\mu=t n$. If $7 \leqq t \leqq 11$ then Lemma 3 implies after a consideration of cases that $k \leqq 6$, and the proof of the theorem for these cases follows from Lemmas 1 and 4.

Theorem 2 is true for groups having $t=13$ or 17 , as well. Lemma 3 also implies (we omit the discussion) that there are only finitely many maximally normal subgroups having at most $t$ parabolic classes, for every $t \geqq 1$.

Call a subgroup of $\Gamma$ cycloidal if it has just one parabolic class (this is Petersson's terminology). Then Corollary 2 also implies

THEOREM 3. The only cycloidal normal subgroups of $\Gamma$ are $\Gamma, \Gamma^{2}, \Gamma^{3}, \Gamma^{\prime}$.

We leave open the question as to whether or not there are only finitely many normal subgroups of $\Gamma$ having a fixed number $t$ of parabolic classes for all positive $t\left(^{(1)}\right.$. The situation for nonnormal subgroups is quite different. Petersson has proved that there are infinitely many cycloidal subgroups, and it is not difficult to extend his result to prove that there are infinitely many subgroups having any fixed number $t$ of parabolic classes. We state this as a theorem.

THEOREM 4. Let $t$ be any integer $>1$. Then there are infinitely many subgroups of $\Gamma$ with just $t$ parabolic classes.

Proof. We first show that for each $t \geqq 1$ there is a subgroup of $\Gamma$ with just $t$ parabolic classes. The commutator subgroup $\Gamma^{\prime}$ has one parabolic class, a representative of which is given by

$$
P=A B A^{-1} B^{-1}
$$

where $A, B$ are defined in (2). Let $\Gamma^{\prime}(t)$ be the subgroup of $\Gamma^{\prime}$ consisting of all words $W$ of $\Gamma^{\prime}$ such that $e_{A}(W) \equiv 0(\bmod t)$. Then $\Gamma^{\prime}(t)$ is a normal subgroup of $\Gamma^{\prime}$ of

(1) This has been answered affirmatively by Leon Greenberg. 
index $t$ in $\Gamma^{\prime}, P$ belongs to $\Gamma^{\prime}(t)$ and therefore the number of parabolic classes of $\Gamma^{\prime}(t)$ is just $t$, according to Theorem 2 of [4]. Since $\Gamma^{\prime}(t)$ is free the genus of $\Gamma^{\prime}(t)$ is just

$$
1+\mu / 12-t / 2=1 \text {, }
$$

since $\mu=6 t$. Furthermore $\Gamma^{\prime}(t)$ is of rank $t+1$ and is freely generated by elements

$$
A_{1}, B_{1}, P_{1}, P_{2}, \ldots, P_{t-1}
$$

such that

$$
P_{t}=A_{1} B_{1} A_{1}^{-1} B_{1}^{-1} P_{1} P_{2} \cdots P_{t-1}
$$

and $P_{1}, P_{2}, \ldots, P_{t}$ are representatives of the different parabolic classes.

Suppose now that $t>1$ and choose any integer $m$ such that $(m, t-1)=1$. Let $\Gamma^{\prime}(t, m)$ be the subgroup of $\Gamma^{\prime}(t)$ consisting of all words of $\Gamma^{\prime}(t)$ whose total exponent sum in $P_{1}, P_{2}, \ldots, P_{t-1}$ is divisible by $m$. Then each $P_{i}, 1 \leqq i \leqq t$ is of exponent $m$ modulo $\Gamma^{\prime}(t, m), \Gamma^{\prime}(t, m)$ is a normal subgroup of $\Gamma^{\prime}(t)$ of index $m$, and it follows from Theorem 2 of [4] that the number of parabolic classes of $\Gamma^{\prime}(t, m)$ is just

$$
m \sum_{i=1}^{t} \frac{1}{m}=t
$$

Since there are arbitrarily many possible choices for $m$, this completes the proof of the theorem.

The corresponding question for the level has a complete answer. There are only finitely many normal subgroups of $\Gamma$ of level $\leqq 5$, but for each $n \geqq 6$ there are infinitely many normal subgroups of that level; for example the groups

$$
\Gamma(n)^{p} \Gamma^{\prime}(n) \Delta\left(S^{n}\right), \quad p=1,2,3, \ldots
$$

We go on now to our first main theorem.

THEOREM 5. Let $\mu=6 q, q$ prime and $>3$. Then if $(q / 3)=1$ there are just two normal subgroups of index $\mu$ (both of genus 1 ) and if $(q / 3)=-1$ there are none.

Proof. We have from the genus formula that

$$
2 g-2=q-6 q / n \text {. }
$$

We know that $g \neq 0$ (since the only normal subgroups of genus 0 are $\Gamma, \Gamma^{2}, \Gamma^{3}, \Gamma(2)$, $\Gamma(3), \Gamma(4), \Gamma(5)$ with indices $1,2,3,6,12,24,60$ respectively) and so $n$ must be even and $\geqq 6$. Thus the only possibilities for $n$ are $n=6,2 q, 6 q$ since $n \mid 6 q$. If $n=6 q$ then $t=1$ and Corollary 2 implies that $6 q \mid 6$, an impossibility. If $n=2 q$ then $t=3$ and Corollary 2 implies that $6 q \mid 12$, again an impossibility. We are left with the possibility $n=6$, when $g=1$. An easy calculation shows that in this case there are no groups when $(q / 3)=-1$ and just two groups when $(q / 3)=1:$ namely, $\left(1, m_{1}, q\right)$ and $\left(1, m_{2}, q\right)$ where $m_{1}, m_{2}$ are the solutions of $m^{2}+m+1 \equiv 0(\bmod q)$ satisfying $0 \leqq m_{1}, m_{2} \leqq q-1$. The proof of the theorem is concluded. 
We go on now to our second main theorem.

THEOREM 6. Let $\mu=12 q, q$ prime and $>11$. Then there are no normal subgroups of index $\mu$.

Proof. The genus formula implies that

$$
g=1+q-6 q / n
$$

Thus $n \mid 6 q$ and $n \geqq 6$ (since $g=0$ can be excluded as in the proof of the previous theorem) and the possibilities can be summarized as follows:

$\begin{array}{rrrrrr}n & 6 & q & 2 q & 3 q & 6 q \\ t & 2 q & 12 & 6 & 4 & 2\end{array}$

If $t=2$ then Corollary 2 implies that $6 q \mid 6$, an impossibility. If $t=4$ then Corollary 2 implies that $3 q \mid 24$, an impossibility. If $t=6$ then Corollary 2 implies that $2 q \mid 720$, again an impossibility since $q>11$. If $t=12$ and $n=q$ then Corollary 1 may be used (since $q>11$ ) and implies that $q \mid 6$, an impossibility. Finally if $t=2 q$ and $n=6$ then $g=1$ and a brief calculation shows that there are no subgroups of genus 1 with $t=2 q$. This concludes the proof.

Once a value of $\mu$ is known for which there are no normal subgroups of $\Gamma$, infinitely many more can be found by the following theorem:

THEOREM 7. Let $G$ be an arbitrary group such that $G$ contains no normal subgroup of index $m$. Let $p$ be a prime $>m$. Then $G$ contains no normal subgroup of index $p^{k} m$, for all positive integers $k$.

Proof. Suppose the contrary. Then $G$ contains a normal subgroup $N$ such that $G / N$ is of order $p^{k} m$. Since $p$ is a prime the first Sylow theorem $\left({ }^{2}\right)$ implies that $G / N$ contains a subgroup of order $p^{k}$; say $G_{1} / N$. Thus $G \supset G_{1} \supset N$, where $\left(G: G_{1}\right)=m$, $\left(G_{1}: N\right)=p^{k}$. Since $p>m$, the second and third Sylow theorems now imply that the number of conjugates of $G_{1} / N$ in $G / N$ is of the form $1+c p, c \geqq 0$ and is a divisor of $m$. Thus $c=0, G_{1} / N$ is a normal subgroup of $G / N$, and hence $G_{1}$ is a normal subgroup of $G$. This is a contradiction since $\left(G: G_{1}\right)=m$. The proof of the theorem is concluded.

The theorem implies for example that there are no normal subgroups of $\Gamma$ of index $30 p^{k}$ for all primes $p>30$, since $\Gamma$ does not contain a normal subgroup of index 30 .

Using Theorem 5 and the lemma below we will prove that there are no normal subgroups of $\Gamma$ with $q$ parabolic classes, where $q$ is a prime such that $(q / 3)=-1$.

LEMMA 5. Suppose that $G_{1}, G$ are normal subgroups of $\Gamma$ of finite index such that $G_{1} \supset G, G_{1}$ is of level $n_{1}$ and has $t_{1}$ parabolic classes, $G$ is of level $n$ and has $t$ parabolic classes. Then $n_{1}\left|n, t_{1}\right| t$.

$\left.{ }^{2}\right)$ Leon Greenberg suggested the use of the Sylow theorems here. 
Proof. Put $\left(G_{1}: G\right)=r$. Then $n t=r n_{1} t_{1}$. The fact that $n_{1} \mid n$ is obvious, since $n_{1}$ is the exponent of $S$ modulo $G_{1}$ and $S^{n} \in G \subset G_{1}$. Furthermore $S^{n_{1}} \in G_{1}$ and so $S^{r n_{1}} \in G$, since $G_{1} / G$ is of order $r$. This implies that $n \mid r n_{1}$, since $n$ is the exponent of $S$ modulo $G$. Hence $t=\left(r n_{1} / n\right) t_{1}$ and so $t_{1} \mid t$. This concludes the proof of the lemma.

We go on to the theorem $\left({ }^{3}\right)$.

THEOREM 8. There are no normal subgroups of $\Gamma$ with $q$ parabolic classes, where $q$ is a prime such that $(q / 3)=-1$.

Proof. Suppose the contrary. Then Lemma 3 implies that there is a normal subgroup $G$ of $\Gamma$ of level $k$ and with $q$ parabolic classes, where $k \leqq q$. Since there are no normal subgroups of $\Gamma$ of index $q^{2}$, we must have $k<q$. By the first Sylow theorem $\Gamma / G$ contains a subgroup of order $q$, say $G_{1} / G$. By the second and third Sylow theorems and the fact that $k<q, G_{1} / G$ must be a normal subgroup of $\Gamma / G$. It follows that $G_{1}$ is a normal subgroup of $\Gamma$ such that $\left(\Gamma: G_{1}\right)=k$.

Now suppose that $G_{1}$ is of level $k_{1}$ and has $q_{1}$ parabolic classes. Then Lemma 5 implies that $k_{1}\left|k, q_{1}\right| q$. Hence $q_{1}=1$ or $q$. The latter is impossible since $k_{1} q_{1}=k$ and $k<q$. Hence $q_{1}=1$. By Theorem $3 G_{1}$ can only be $\Gamma, \Gamma^{2}, \Gamma^{3}$ or $\Gamma^{\prime}$. Since $G \supset G_{1}^{\prime}$ $\left(G_{1} / G\right.$ is cyclic and hence abelian) the first three possibilities can be eliminated, in virtue of the information below:

$\begin{array}{lllll} & \mu & n & t & g \\ \Gamma & 1 & 1 & 1 & 0 \\ \Gamma^{2} & 2 & 2 & 1 & 0 \\ \Gamma^{3} & 3 & 3 & 1 & 0 \\ \Gamma^{\prime} & 6 & 6 & 1 & 1 \\ \left(\Gamma^{2}\right)^{\prime} & 18 & 6 & 3 & 1 \\ \left(\Gamma^{3}\right)^{\prime} & 24 & 6 & 4 & 1\end{array}$

This leaves only the possibility $G_{1}=\Gamma^{\prime}$. In this case $G$ must be of level 6 and genus 1 . Theorem 5 now implies that no such group exists, since $(q / 3)=-1$. This concludes the proof of the theorem.

4. The enumeration of normal subgroups for small indices. In this section we determine all normal subgroups of $\Gamma$ of indices $\leqq 66$. The values of $N(\mu)$ so obtained are listed below:

$$
\begin{array}{rrrrrrrrrrrrrrr}
\mu & 1 & 2 & 3 & 6 & 12 & 18 & 24 & 30 & 36 & 42 & 48 & 54 & 60 & 66 \\
N(\mu) & 1 & 1 & 1 & 2 & 1 & 1 & 2 & 0 & 0 & 2 & 2 & 1 & 1 & 0
\end{array}
$$

The groups themselves are given by the following theorem:

( $\left.{ }^{3}\right)$ Leon Greenberg has given a different proof of this theorem. 
THEOREM 9. The normal subgroups $G$ of $\Gamma$ of indices $\leqq 66$ are just those given in the following table:

(10)

$\begin{array}{rccc}\mu & G & \mu & G \\ 1 & \Gamma & 30 & \text { none } \\ 2 & \Gamma^{2} & 36 & \text { none } \\ 3 & \Gamma^{3} & 42 & (1,2,7),(1,4,7) \\ 6 & \Gamma(2), \Gamma^{\prime} & 48 & G_{4,2}, G_{3,4} \\ 12 & \Gamma(3) & 54 & (3,0,1) \\ 18 & (1,1,3) & 60 & \Gamma(5) \\ 24 & \Gamma(4),(2,0,1) & 66 & \text { none }\end{array}$

Proof. The cases $\mu=1,2,3,6$ offer no difficulty. If $\mu=12$ then $n=3, t=4, g=0$ (giving $G=\Gamma(3)$ ) or else $n=6, t=2, g=1$ which is impossible by our knowledge of normal subgroups of genus 1 . If $\mu=18$ then $n=6, t=3, g=1$ (giving $G=(1,1,3)$ ) or else $n=18, t=1, g=2$ which is impossible by Corollary 2 . If $\mu=24$ then $n=4$, $t=6, g=0$ (giving $G=\Gamma(4)$ ), or $n=6, t=4, g=1$ (giving $G=(2,0,1)$ ) or else $n=12$, $t=2, g=2$ which is impossible by Corollary 2 . There are no subgroups of index 30 , by Theorem 5 . If $\mu=36$ then $n=6, t=3, g=1$ or $n=9, t=2, g=2$ or $n=18, t=1$, $g=3$ all of which are impossible by our knowledge of normal subgroups of genus 1 and Corollary 2. The case $\mu=42$ is covered by Theorem 5 . We put aside the case $\mu=48$ for the moment. If $\mu=54$ then $n=6, t=9, g=1$ (giving $G=(3,0,1)$ ) or $n=18$, $t=3, g=4, n=54, t=1, g=5$ both of which are impossible by Corollary 2 . We put aside the case $\mu=60$ for the moment. The case $\mu=66$ is covered by Theorem 5 .

We have left therefore the cases $\mu=48, \mu=60$. If $\mu=60$ then $n=5, t=12, g=0$ (giving $G=\Gamma(5)$ ) or $n=6, t=10, g=1, n=10, t=6, g=3, n=15, t=4, g=4, n=30$, $t=2, g=5$. All but the second of these are immediately excluded by our knowledge of normal subgroups of genus 1 and Corollary 2. Assuming that $n=10, t=6, g=3$ we find that the possible values of the integer $k$ defined in Lemma 3 are $k=1,2,5$. The first two values are excluded by Lemma 1 , since $n$ must be a divisor of 6 in these cases, and $n=10$. The last value of $k$ is excluded by Lemma 3 , since there is no normal subgroup of index 30 .

There remains only the case $\mu=48$. The possibilities here are $n=6, t=8, g=1$, $n=8, t=6, g=2, n=12, t=4, g=3, n=24, t=2, g=4$. Of these the first and the last can be immediately excluded as before. The second implies after some calculation that $G \supset \Delta\left(S^{8}, T S^{4} T S^{-4}\right)=G_{4,2}$. The third implies similarly that

$$
G \supset \Delta\left(S^{12}, T S^{3} T S^{-3}\right)=G_{3,4}
$$


or that $G \supset \Delta\left(S^{12}, T S^{4} T S^{-4}\right)=G_{4,3}$. Since $G_{4,3}$ is of index 72 in $\Gamma$ the latter is impossible, leaving only $G \supset G_{4,2}$ or $G \supset G_{3,4}$. Since $G, G_{4,2}, G_{3,4}$ are all of index 48 in $\Gamma$ this implies that $G=G_{4,2}$ or $G=G_{3,4}$.

This completes the proof.

We remark in conclusion that the function $N(\mu)$ does become arbitrarily large. It was shown in [4] that if $\xi(\mu)$ denotes the number of normal subgroups of $\Gamma$ of genus 1 and index $6 \mu$, and $q$ is a prime $\equiv 1(\bmod 3)$, then

$$
\xi\left(q^{2 r}\right)=2 r-1 \text {. }
$$

Thus

$$
N\left(6 q^{2 r}\right) \geqq 2 r-1
$$

for all primes $q$ such that $(q / 3)=1$.

\section{REFERENCES}

1. H. S. M. Coxeter and W. O. Moser, Generators and relations for discrete groups, 2 nd ed., Springer, Berlin, 1965.

2. I. M. S. Dey, Schreier systems in free products, Proc. Glasgow Math. Assoc. 7 (1965), 61-79.

3. R. C. Gunning, Lectures on modular forms, Annals of Mathematical Studies, No. 48, Princeton Univ. Press, Princeton, N. J., 1962.

4. M. Newman and M. I. Knopp, Congruence subgroups of positive genus of the modular group, Illinois J. Math. 9 (1965), 577-583.

5. M. Newman, A complete description of the normal subgroups of genus one of the modular group, Amer. J. Math. 86 (1964), 17-24.

6. - Free subgroups and normal subgroups of the modular group, Illinois J. Math. 8 (1964), 262-265.

National Bureau of Standards, WASHington, D. C. 